\title{
Dentifrícios infantis comercializados no município de Londrina/PR, Brasil: análise da rotulagem segundo especificações da Agência Nacional de Vigilância Sanitária
}

\author{
Children's toothpastes commercialized in Londrina/PR, Brazil: \\ labeling analysis according to specifications of the National Health \\ Surveillance Agency
}

\section{Letícia Nascimento Martins da Fonseca (iD)}

\section{Caroline Pagani Martins}

Luciana Tiemi Inagaki (iD

Pablo Guilherme Caldarelli* ii

Universidade Estadual de Londrina (UEL), Londrina, PR, Brasil

\section{* E-mail: pablocaldarelli@uel.br}

Recebido: 03 ago 2020 Aprovado: 19 jan 2021

\section{RESUMO}

Introdução: Devido à grande variedade de formulações químicas entre os dentifrícios infantis e à possibilidade de apresentarem ou não o flúor $(F)$ em sua composição, a vigilância é essencial para o controle da qualidade desses produtos. Objetivo: Analisar a rotulagem e as informações presentes em dentifrícios infantis, disponíveis comercialmente, segundo especificações das Resoluções $n^{\circ}$ 07, de 10 de fevereiro de 2015, e $n^{\circ} 79$, de 28 de agosto de 2000, da Agência Nacional de Vigilância Sanitária (Anvisa). Método: Estudo transversal realizado por meio da análise da disponibilidade, preço e avaliação das embalagens (primárias e secundárias) de dentifrícios infantis à venda no município de Londrina, localizado no Norte do Paraná, Brasil. Os produtos foram adquiridos no mês de maio do ano de 2019. Os dados foram analisados por meio de estatística descritiva. Resultados: Foram avaliados 11 dentifrícios infantis dos quais $73,0 \%$ (oito) apresentaram concentração convencional de $F$ ( $\geq 1.000$ ppm F), 9,0\% (um) baixa concentração de $F$ ( 500 ppm F) e 18,0\% (dois) eram sem $\mathrm{F}$. $\mathrm{O}$ valor do grama do dentifrício sem $\mathrm{F}$ foi cerca de três vezes maior que o dos demais produtos. Todos os dentifrícios analisados atendiam às normas estabelecidas pela Resolução $n^{\circ}$ 79/2000 e 82,0\% (nove) estavam em consonância com as exigências da Resolução $n^{\circ}$ 07/2015. Conclusões: Há uma ampla oferta de dentifrícios infantis no mercado que, em sua grande maioria, encontra-se de acordo com as normas da Anvisa sobre composição e rotulagem. Contudo, ressalta-se a importância da vigilância desses produtos para a garantia do máximo efeito no controle da cárie dentária e o mínimo risco para o desenvolvimento de fluorose dental.

PALAVRAS-CHAVE: Dentifrícios; Fluoretação; Legislação em Saúde; Vigilância Sanitária; Cárie Dentária

\section{ABSTRACT}

Introduction: Due to the wide variety of chemical formulations among children's toothpaste and the possibility of having fluoride in its composition, surveillance is essential for quality control of these products. Objective: To analyze the labeling and recommendations in commercially available children's toothpastes, according to Resolutions No. 07, 10 February, 2015 and No. 79, 28 August, 2000, published by the National Health Surveillance Agency (Anvisa). Method: This is cross-sectional study carried out to analyze the availability, price and packaging (primary and secondary) of children's toothpaste for sale in Londrina, located in the north of Paraná, Brazil. The products were purchased in May of 2019. Data were analyzed using descriptive statistics. Results: Eleven children's toothpastes were evaluated and $73.0 \%$ (8) had conventional fluoride concentration ( $\geq 1,000 \mathrm{ppm} \mathrm{F}$ ), 9.0\% (1) low fluoride concentration (500 ppm F) and $18.0 \%$ (2) did not have fluoride. The gram value of fluoride-free toothpaste was about 3 times greater than the other products. All dentifrices analyzed met the standards determined 
by Resolution No. 79/2000 and $82.0 \%$ (9) were in line with the requirements of Resolution No. 07/2015. Conclusions: There was a wide offer of children's toothpaste on the market and, the most part of these toothpastes was in accordance with Anvisa's rules concerning composition and labeling. However, it is important to emphasize the importance to have a frequently monitoring of these products to keep an effective dental caries control and to guarantee the minimum risk for dental fluorosis.

KEYWORDS: Toothpastes; Fluoridation; Health Legislation; Health Surveillance; Dental Caries

\section{INTRODUÇÃO}

Os dentifrícios são formulados com uma diversidade de componentes, como: sistemas abrasivos, detergentes, flavorizantes, solventes, umectantes, aglutinantes, edulcorantes e conservantes ${ }^{1}$. Apesar de terem sido rotulados como cosméticos no passado, atualmente, os dentifrícios têm assumido um papel terapêutico importante, principalmente no controle da cárie dentária. Nesse sentido, a escovação com dentifrícios fluoretados tem sido apontada como um dos principais fatores responsáveis pelo declínio da cárie dentária em países desenvolvidos e em desenvolvimento, como o Brasil ${ }^{2}$. Isso tem sido atribuído ao fluoreto agregado às formulações, que age reduzindo os processos de desmineralização e ativando a remineralização das estruturas dentais ${ }^{3}$. Todavia, segundo a Agência Nacional de Vigilância Sanitária (Anvisa), esses produtos ainda permanecem vinculados à categoria de produtos de higiene pessoal, cosméticos e perfumes ${ }^{4}$.

Diferentes formulações químicas de dentifrícios estão disponíveis no mercado, dentre as quais se encontram aqueles direcionados ao público infantil, que podem apresentar ou não o $\mathrm{F}$ em suas composições. As concentrações de $F$, quando presentes nesses dentifrícios, também se mostram bastante variadas ${ }^{5}$. Nesse sentido, revisões sistemáticas da literatura têm concluído que esses produtos devem conter uma concentração de pelo menos 1.000 ppm de flúor $(F)$ para terem efeito no controle da cárie dentária e que essa concentração deve estar em forma quimicamente solúvel para que o fluoreto possa interferir de modo efetivo no processo de desmineralização e remineralização da superfície dentária ${ }^{6,7,8}$. Especificamente em relação à dentição decídua, revisões sistemáticas que incluíram apenas ensaios clínicos com pré-escolares encontraram evidências para redução significativa no incremento de cárie a partir de $1.000 \mathrm{ppm} F$, quando comparados aos dentifrícios sem $\mathrm{F}$ ou com menos de $600 \mathrm{ppm} \mathrm{F}^{7,8}$.

Diante desses aspectos, a vigilância dos dentifrícios torna-se cada vez mais importante para o controle da qualidade dos produtos disponíveis comercialmente e daqueles distribuídos pelos serviços públicos de saúde bucal no Brasil ${ }^{7,8,9}$. Para isso, atualmente encontram-se vigentes Resoluções da Anvisa ${ }^{4,10}$ que regulamentam os produtos para higiene bucal, dentre eles os dentifrícios. Em relação à concentração de $\mathrm{F}$, a Resolução $\mathrm{n}^{\circ} 79$, publicada em 28 de agosto de 2000 pela Anvisa ${ }^{10}$, determina apenas a concentração máxima total presente nas formulações, que não deve exceder $0,15 \%$ expresso em $F(1.500$ ppm F) e também especifica os diversos sais de $\mathrm{F}$ que podem ser utilizados em dentifrícios. Além disso, exige que no rótulo dos dentifrícios estejam claramente expressos o nome e a concentração do composto de
F adicionado, bem como o modo de uso. Entretanto, essa Resolução não informa quanto de flúor solúvel (potencialmente ativo contra cárie) um dentifrício deveria conter e manter ao longo do seu prazo de validade ${ }^{10}$.

Quanto à definição, classificação, aos requisitos técnicos de rotulagem e procedimento eletrônico para regularização de produtos de higiene pessoal, cosméticos e perfumes, a Anvisa publicou a Resolução $\mathrm{n}^{\circ}$ 7, em 10 de fevereiro de 20154. Essa Resolução define todas as indicações necessárias referentes aos produtos e também as informações indispensáveis que devem figurar nos rótulos concernentes a sua utilização, como: a descrição dos componentes da fórmula por meio de uma designação genérica, a identificação precisa da empresa fabricante, o período de tempo em que o produto consegue manter suas propriedades se utilizado e armazenado da maneira adequada e o número de registro da mercadoria para comprovar a sua autorização de comercialização ${ }^{4}$. Essa normativa foi alterada pela Resolução $\mathrm{n}^{\circ} 288$, de 4 de junho de 2019, tendo-se, contudo, poucas modificações no que se refere aos componentes dos invólucros ${ }^{11}$.

Dessa forma, considerando a importância de se equilibrar benefícios e riscos no uso de dentifrícios e a necessidade de que os rótulos desses produtos contenham informações claras e seguras sobre as suas características, o objetivo do presente estudo foi analisar a rotulagem e as informações presentes em dentifrícios infantis disponíveis comercialmente, verificando a sua conformidade com as especificações estabelecidas pelas Resoluções $n^{\circ}$ $7 / 2015^{4}$ e $n^{\circ} 79 / 2000^{10}$, publicadas pela Anvisa.

\section{MÉTODO}

Trata-se de um estudo transversal realizado por meio da análise de disponibilidade, preço e avaliação das embalagens de dentifrícios infantis comercializados no município de Londrina, localizado na região Norte do estado do Paraná, Brasil. Para isso, cinco estabelecimentos comerciais de grande porte foram visitados para levantamento inicial dos dentifrícios disponíveis para venda. Com base nesse levantamento, foi selecionado para a realização da compra o estabelecimento com a maior variedade de dentifrícios infantis, tratando-se de um supermercado. Ainda nesse aspecto, optou-se por adquirir todos os produtos em um local específico para possibilitar a análise da precificação, evitando variações relativas às margens de lucro empregadas pelos diferentes comércios. Os produtos foram adquiridos no mês de maio do ano de 2019, sendo a amostra composta por um total de 11 dentifrícios infantis. 
Os dados foram coletados a partir da análise da rotulagem dos produtos de embalagens primárias e secundárias. Conforme a Anvisa, entende-se como embalagem primária o envoltório ou recipiente que se encontra em contato direto com os produtos, e embalagem secundária aquela destinada a conter a(s) embalagem(ns) primária(s) ${ }^{4}$. Para garantir o sigilo das marcas/ nomes comerciais dos dentifrícios analisados neste estudo, foi adotada uma codificação alfanumérica, utilizando a letra $D$ (dentifrício) seguida da numeração correspondente à ordem de análise (D01 até D11).

Para a coleta, considerou-se os critérios descritos na Resolução $n^{\circ}$ 7/20154, descritos no Quadro 1, e na Resolução no 79/200010, ambas publicadas pela Anvisa. Para organização e tabulação dos dados foram construídos dois instrumentos. 0 primeiro instrumento foi composto por informações relativas ao nome do produto/conteúdo, à marca do produto, ao prazo de validade, ao modo de uso, à concentração e tipo de F/abrasivo e ao preço do produto/peso (g). No segundo instrumento foram abordadas as recomendações e precauções presentes nas embalagens dos dentifrícios analisados. A extração dos dados das embalagens foi realizada de forma sequencial por produto, conduzida por um único pesquisador, previamente treinado.

Os dados foram analisados por meio de estatística descritiva, valores absolutos e percentuais, verificando o cumprimento dos principais requisitos nas embalagens primárias e secundárias dos produtos. Para isso, utilizou-se o software Microsoft Excel $®$.

\section{RESULTADOS E DISCUSSÃO}

A Resolução $n^{\circ} 7 / 2015^{4}$ da Anvisa tem como objetivo estabelecer as informações indispensáveis que devem figurar nos rótulos dos produtos de higiene pessoal, cosméticos e perfumes, concernentes a sua utilização, assim como toda a indicação necessária referente ao produto, tanto em embalagens primárias como em secundárias, determinando que contenham informações e advertências referentes ao produto conforme legislação vigente. Em junho de 2019, porém, instituiu-se a Resolução $n^{\circ}$ 288 da Anvisa ${ }^{11}$, alterando alguns aspectos da normativa anterior. Especificamente sobre a arte das embalagens, acrescentou-se a exigência de que as informações sejam legíveis e que os produtos importados cujos rótulos originais não contenham os dados requeridos pelas normas brasileiras sejam readequados previamente à sua comercialização.

Nesse sentido, dos 11 dentifrícios analisados neste estudo, dois apresentavam apenas embalagens primárias, enquanto os outros nove possuíam embalagem primária e secundária. Em todas as embalagens foram encontradas as informações referentes ao lote ou partida, número de registro do produto, país de origem e dados do fabricante, conforme a recomendação da Resolução.

No Quadro 2 são apresentados os dados coletados por meio das embalagens, organizados de acordo com a codificação do produto, ingredientes/composição, prazo de validade, modo de uso, concentração em ppm F, tipo de F/abrasivo e custo do produto (preço/peso).

De acordo com a legislação vigente, nas embalagens devem estar contidas informações sobre as precauções e recomendações a serem respeitadas no que tange a utilização dos dentifrícios. No Quadro 3 são apresentadas as recomendações encontradas em cada uma das embalagens analisadas. Todos os dentifrícios alertavam sobre a não ingestão do produto e a necessidade de que este fosse aplicado por adulto ou sob sua supervisão. Em oito $(73,0 \%)$ havia recomendações sobre seguir as orientações de um médico ou dentista nos casos em que há a utilização de $F$ proveniente de outras fontes, manter fora do alcance de crianças e sobre a quantidade a ser utilizada por crianças de até seis anos de idade. Dois $(18,0 \%)$ alertavam a não usar o dentifrício caso a embalagem estivesse aberta e que o produto não continha açúcar em sua composição e apenas um $(9,0 \%)$ orientava a suspender o uso em caso de reação indesejável. Observou-se, desse modo, a ausência de padronização entre as informações fornecidas pelos invólucros dos diferentes dentifrícios avaliados.

Quadro 1. Itens da rotulagem obrigatória segundo a Resolução $n^{\circ}$ 7, de 10 de fevereiro de 2015.

\begin{tabular}{|c|c|c|}
\hline & Item & Embalagem \\
\hline 1 & Nome do produto e grupo/tipo a que pertence no caso de não estar implícito no nome & Primária e Secundária \\
\hline 2 & Marca & Pecundária \\
\hline 3 & Número de registro do produto & Primária \\
\hline 4 & Lote ou partida & Secundária \\
\hline 5 & Conteúdo de validade & Secundária \\
\hline 6 & Pabricante/Importador/Titular & Secundária \\
\hline 7 & Domicílio do fabricante/Importador/Titular & Secundária \\
\hline 8 & Modo de uso (se for o caso) & Primária ou Secundária \\
\hline 9 & Advertências e restrições de uso (se for o caso) & Primária e Secundária \\
\hline 10 & Rotulagem específica & Primária e Secundária \\
\hline 11 & Ingredientes/Composição & Secundária \\
\hline 12 & &
\end{tabular}

Fonte: Resolução $n^{\circ} 7 / 2015^{4}$. 
Quadro 2. Informações presentes nas embalagens primárias e secundárias de dentifrícios infantis analisados quanto à data de fabricação e validade, à composição, ao modo de uso, à concentração e ao tipo de F/abrasivo e preço/peso em gramas. Londrina/PR, 2019.

\begin{tabular}{|c|c|c|c|c|c|}
\hline 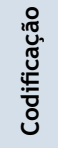 & Data fabricação/ validade & $\begin{array}{l}\text { Ingrediente/ } \\
\text { Composição }\end{array}$ & Modo de uso & $\begin{array}{c}\text { Concentração }(\mathrm{ppm} F) \text { e } \\
\text { tipo de F/abrasivo }\end{array}$ & $\begin{array}{c}\text { Preço (R\$)/ } \\
\text { Peso (g) }\end{array}$ \\
\hline D01 & $\begin{array}{l}\text { Sem data de fabricação, } \\
\text { apenas validade }\end{array}$ & Presente & Presente & Sem Flúor/Sílica Hidratada & $\mathrm{R} \$ 16,90 / 50 \mathrm{~g}$ \\
\hline D02 & $\begin{array}{l}\text { Sem data de fabricação, } \\
\text { apenas validade }\end{array}$ & Presente & Presente & Sem Flúor/Sílica Hidratada & $\mathrm{R} \$ 3,98 / 50 \mathrm{~g}$ \\
\hline D03 & $\begin{array}{l}\text { Sem data de fabricação, } \\
\text { apenas validade }\end{array}$ & Presente & Ausente & $500 \mathrm{NaF} /$ Sílica Hidratada & $\mathrm{R} \$ 3,98 / 50 \mathrm{~g}$ \\
\hline D04 & $\begin{array}{l}\text { Sem data de fabricação, } \\
\text { apenas validade }\end{array}$ & Presente & Ausente & 1.100 NaF/Sílica Hidratada & $\mathrm{R} \$ 3,98 / 50 \mathrm{~g}$ \\
\hline D05 & $\begin{array}{l}\text { Sem data de fabricação, } \\
\text { apenas validade }\end{array}$ & Presente & Presente & 1.100 NaF/Sílica Hidratada & $\mathrm{R} \$ 10,40 / 100 \mathrm{~g}$ \\
\hline D06 & $\begin{array}{l}\text { Sem data de fabricação, } \\
\text { apenas validade }\end{array}$ & Presente & Presente & 1.100 NaF/Sílica Hidratada & $\mathrm{R} \$ 5,59 / 50 \mathrm{~g}$ \\
\hline D07 & $\begin{array}{c}\text { Apresenta data de fabricação } \\
\text { e de validade }\end{array}$ & Presente & Presente & 1.100 NaF/Sílica & $\mathrm{R} \$ 4,69 / 50 \mathrm{~g}$ \\
\hline D08 & $\begin{array}{l}\text { Sem data de fabricação, } \\
\text { apenas validade }\end{array}$ & Presente & Presente & 1.100 NaF/Sílica Hidratada & $\mathrm{R} \$ 5,49 / 50 \mathrm{~g}$ \\
\hline D09 & $\begin{array}{c}\text { Apresenta data de fabricação } \\
\text { e de validade }\end{array}$ & Presente & Presente & 1.100 NaF/Sílica Hidratada & $\mathrm{R} \$ 9,69 / 100 \mathrm{~g}$ \\
\hline D10 & $\begin{array}{l}\text { Sem data de fabricação, } \\
\text { apenas validade }\end{array}$ & Presente & Presente & 1.100 NaF/Sílica Hidratada & $\mathrm{R} \$ 5,59 / 130 \mathrm{~g}$ \\
\hline D11 & $\begin{array}{l}\text { Sem data de fabricação, } \\
\text { apenas validade }\end{array}$ & Presente & Presente & $\begin{array}{l}\text { 1.100 MFP/Carbonato de } \\
\text { Cálcio, Sílica Hidratada }\end{array}$ & $\mathrm{R} \$ 4,84 / 50 \mathrm{~g}$ \\
\hline
\end{tabular}

Fonte: Elaborado pelos autores, 2020.

Quadro 3. Recomendações/precauções presentes em embalagens primárias e secundárias dos dentifrícios analisados no estudo. Londrina/PR, 2019.

\begin{tabular}{|c|c|c|c|c|c|c|c|c|}
\hline \multirow{2}{*}{ 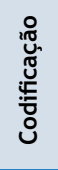 } & \multicolumn{8}{|c|}{ Recomendação/Precaução } \\
\hline & $\begin{array}{l}\text { Quantidade } \\
\text { adequada para } \\
\text { crianças de } \\
\text { até } 6 \text { anos }\end{array}$ & Não ingerir & $\begin{array}{l}\text { Aplicar sob a } \\
\text { supervisão de } \\
\text { um adulto }\end{array}$ & $\begin{array}{c}\text { Usar sob } \\
\text { orientação } \\
\text { de médico/ } \\
\text { dentista }\end{array}$ & $\begin{array}{l}\text { Manter fora } \\
\text { do alcance de } \\
\text { crianças }\end{array}$ & $\begin{array}{l}\text { Não usar caso } \\
\text { a embalagem } \\
\text { esteja violada }\end{array}$ & $\begin{array}{l}\text { Não contém } \\
\text { açúcar }\end{array}$ & $\begin{array}{l}\text { Suspender } \\
\text { uso em caso } \\
\text { de reação } \\
\text { indesejável }\end{array}$ \\
\hline D01 & - & $x$ & $x$ & - & $x$ & $\mathrm{x}$ & - & - \\
\hline D02 & $x$ & $x$ & $x$ & - & $x$ & - & - & - \\
\hline D03 & $x$ & $x$ & $x$ & $x$ & $x$ & - & - & - \\
\hline D04 & $x$ & $x$ & $\mathrm{x}$ & $x$ & $x$ & - & - & - \\
\hline D05 & $x$ & $x$ & $\mathrm{x}$ & $x$ & $x$ & $\mathrm{x}$ & - & - \\
\hline D06 & $x$ & $x$ & $x$ & $x$ & $x$ & - & - & - \\
\hline D07 & $x$ & $x$ & $x$ & $x$ & - & - & - & - \\
\hline D08 & $x$ & $x$ & $x$ & $X$ & $x$ & - & $X$ & - \\
\hline D09 & - & $x$ & $x$ & - & - & - & $X$ & - \\
\hline D10 & - & $x$ & $x$ & $x$ & $x$ & - & - & $x$ \\
\hline D11 & $x$ & $x$ & $x$ & $x$ & $=$ & - & - & - \\
\hline
\end{tabular}

Fonte: Elaborado pelos autores, 2020.

A absorção excessiva de $\mathrm{F}$ durante o período de formação dos dentes, especialmente para crianças até seis anos de idade, pode levar a alterações na mineralização das estruturas dentais, conhecida como fluorose dental. Assim, a deglutição e consequente ingestão/ absorção inadvertida de $\mathrm{F}$ por crianças por meio dos dentifrícios fluoretados são fatores de risco para a fluorose ${ }^{12,13}$. Quanto mais jovem a criança, mais propensa ela estará a deglutir o produto, provocando uma ingestão acima da estimativa empírica de dose de ingestão diária de $\mathrm{F}$ que mantém a fluorose em graus que não causam comprometimento estético $(0,05$ a $0,07 \mathrm{mg} \mathrm{F} / \mathrm{kg}$ peso corpóreo/dia $)^{12}$. Contudo, ressalta-se que, nas normativas vigentes, como a Resolução da Anvisa $n^{\circ} 16$, de 12 de abril $2011^{14}$, a qual dispõe sobre as advertências obrigatoriamente presentes em produtos que contenham substâncias de uso restrito, não há nenhuma exigência quanto a apresentação de alertas sobre o potencial de toxicidade dos dentifrícios fluoretados. 
Enfatiza-se, portanto, a necessidade da supervisão das crianças durante a escovação, tendo em vista que, devido à pouca idade, podem apresentar dificuldades motoras para expelir toda a saliva com o dentifrício. Desse modo, é possível se obter o máximo efeito no controle da cárie dentária e o mínimo risco de desenvolvimento de fluorose ${ }^{13}$. Nesse contexto, é essencial que conste, nas embalagens dos dentifrícios, informações que orientem os adultos responsáveis sobre o modo adequado de uso do produto, identificando a quantidade a ser utilizada, a frequência de uso e ainda alertas sobre a deglutição e a ingestão de $F$, o que não foi identificado em dois $(18,0 \%)$ dos produtos analisados neste estudo.

Para Batista e Valença ${ }^{15}$, os cuidados e as recomendações pertinentes ao uso do $F$ não se restringem apenas a pais/responsáveis e crianças. Os dentistas necessitam ter maior cuidado na identificação das fontes de consumo de $F$ empregadas por seus pacientes, orientando os responsáveis pela criança sobre as precauções a serem consideradas ao se proceder com a escovação dental. É relevante, ainda, a orientação a outros profissionais de saúde quanto ao risco da suplementação de flúor e quanto à indicação correta desse método, tendo em vista que suplementos ainda têm sido prescritos mesmo sabendo-se que esta indicação não está baseada nas evidências científicas que explicam o mecanismo de ação do fluoreto no controle da cárie dentária ${ }^{15,16}$.

Com relação à concentração de $\mathrm{F}$ nos dentifrícios avaliados, oito $(73,0 \%)$ continham 1.100 ppm F, um $(9,0 \%) 500$ ppm $F$ e dois (18,0\%) eram sem F. A concentração máxima de $F$ adicionada aos dentifrícios convencionais no Brasil é de $1.500 \mathrm{ppm}$ de $F$, seguindo as normas regulamentadas pela Resolução ${ }^{\circ} 79 / 2000$ da Anvisa, que determinam o valor máximo de $0,15 \%$ de $\mathrm{F}(1.500 \mathrm{ppm}$ de $F)^{10}$. Dessa forma, todos os dentifrícios avaliados nesse estudo se encontram de acordo com essa resolução. Contudo, revisões sistemáticas da literatura mundial, que confirmaram a eficácia e os benefícios do uso dos dentifrícios fluoretados no controle da cárie dentária, quando comparados a placebos, apontam que, para se garantir o efeito preventivo de maneira significativa, os dentifrícios devem conter em sua composição uma concentração mínima de 1.000 ppm de $F^{6,7,8}$, o qual, por princípio, deve estar em sua forma solúvel.

De acordo com Santos et al. ${ }^{7}$, dentifrícios com concentrações iguais ou maiores que 1.000 ppm $\mathrm{F}$ possuem efeito anticárie, enquanto dentifrícios com menos de 600 ppm $\mathrm{F}$ não produzem redução significativa na incidência da doença, quando comparados a um dentifrício placebo. Nessa discussão, torna-se importante ressaltar também que ainda não existem dados clínicos disponíveis que mostrem que os dentifrícios com baixa concentração de $F$, independentemente da formulação, sejam eficazes na redução dos riscos de desenvolvimento de fluorose dental em crianças em idade pré-escolar ${ }^{7}$. Considerando que muitas crianças brasileiras ainda são acometidas por cárie na primeira infância, o uso regular de dentifrícios fluoretados é fundamental no controle dessa doença. Assim sendo, aqueles cuja concentração de $F$ é baixa ou os que não contém $F$ não devem ser indicados ${ }^{17}$.
Ainda com relação à Resolução $n^{\circ} 79 / 2000^{10}$, destaca-se que parâmetros mínimos de $\mathrm{F}$ até o prazo de validade dos produtos não estão contemplados na referida norma da Anvisa. Nesse sentido, tem sido ratificada a necessidade de revisão dessa Resolução, estabelecendo a concentração mínima de $\mathrm{F}$ solúvel que um dentifrício deveria manter por determinado tempo após sua fabricação para o seu máximo efeito no controle da cárie dentária ${ }^{6}$. Contudo, essa discussão não é uma especificidade do Brasil, mas sim um problema mundial sinalizado pela Federação Dentária Internacional $(\mathrm{FDI})^{18}$.

Outro aspecto relevante diz respeito à combinação adequada do tipo de composto fluoretado e sistema abrasivo, a qual é fundamental para garantir que a formulação tenha eficácia anticárie (flúor solúvel). Diante disso, dentifrícios contendo cálcio $\left(\mathrm{Ca}^{++}\right)$ no abrasivo não podem ser formulados com compostos fluoretados que geram íon fluoreto $\left(\mathrm{F}^{-}\right)$quando dissolvidos e agregados à formulação $\left(\mathrm{NaF}, \mathrm{SnF}_{2} \text { ou } \mathrm{AmF}\right)^{19}$. O F' reage imediatamente com íons $\mathrm{Ca}^{++}$do abrasivo formando dentro da bisnaga do dentifrício o Fluoreto de cálcio $\left(\mathrm{CaF}_{2}\right)$, o qual é insolúvel e não tem efeito no controle da cárie dentária. Dessa forma, a sílica $\left(\mathrm{SiO}_{2}\right)$ tem sido usada como agente abrasivo em dentifrícios que geram íon $\mathrm{F}$, como observado nos resultados apresentados no Quadro 2. Essa combinação permite que todo o $\mathrm{F}$ agregado permaneça solúvel na formulação pelo prazo de validade ${ }^{20}$, agindo no controle das lesões de cárie.

Nesse contexto, considerando que as diferentes combinações de compostos fluoretados com sistemas abrasivos podem influenciar a concentração de $\mathrm{F}$ nos dentifrícios ao longo do tempo, as informações relacionadas à data de fabricação e aos prazos de validade dos produtos tornam-se relevantes, embora a Resolução da Anvisa recomende apenas a informação do prazo de validade (Quadro 1).

Em relação aos resultados relativos ao custo por quantidade em gramas de produto, oito $(73,0 \%)$ dentifrícios apresentaram embalagens com peso igual a 50 gramas, dois $(18,0 \%)$ continham 100 gramas e um $(9,0 \%)$ possuía 130 gramas. Os preços dos produtos variaram entre $R \$ 3,98$ e $R \$ 16,90$ a unidade. A Figura apresenta o valor do grama de cada um dos produtos analisados, o qual variou de $\mathrm{R} \$ 0,04$ a $\mathrm{R} \$ 0,35$ o grama.

De acordo com os achados, o dentifrício com o valor mais elevado era não fluoretado, apresentando um valor de R\$0,34 por grama. Em contrapartida, o mais barato, que era fluoretado, apresentou o valor de R\$0,04 por grama. Quando comparadas as médias de preços de dentifrícios com e sem $\mathrm{F}$, observou-se que o grama do dentifrício sem flúor foi três vezes maior do que os com flúor. Esse dado se encontra de acordo com outros estudos, como o de Roza et al. ${ }^{21}$, que avaliaram as características dos dentifrícios infantis disponíveis no mercado brasileiro de acordo com sua apresentação comercial, disponibilidade e preços. Os autores concluíram que há uma grande variação de preço entre esses produtos, sendo que os sem $\mathrm{F}$ são os mais caros.

Nesse sentido, os consumidores devem se atentar à relação entre o peso e o preço dos dentifrícios infantis, bem como o 


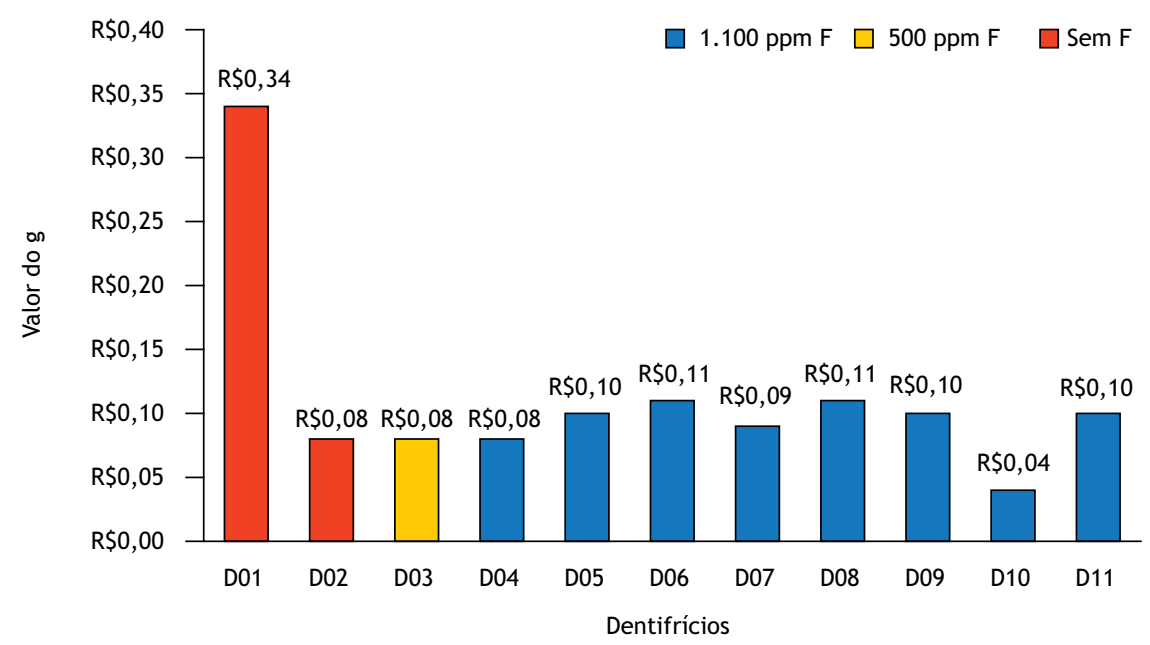

Fonte: Elaborada pelos autores, 2020.

Figura. Custo (por grama) dos dentifrícios infantis analisados no estudo. Londrina/PR, 2019.

custo-benefício, tendo em vista que há dentifrícios fluoretados produzidos conforme os padrões preconizados pelas evidências científicas disponíveis com preços, inclusive, menores do que aqueles que não possuem eficácia cientificamente comprovada ${ }^{21}$. Além disso, ainda que eficaz o dentifrício com $\mathrm{F}$ é, em grande parte, dependente de sua integração aos hábitos pessoais de higiene bucal, à disponibilidade e à capacidade dos indivíduos em adquiri-los e usá-los regularmente ${ }^{22}$.

Por fim, ressalta-se que, embora o quantitativo de dentifrícios infantis fluoretados analisados nesta pesquisa não represente a totalidade de produtos disponíveis para comercialização no mercado brasileiro, os resultados encontrados podem contribuir para que novos estudos sejam conduzidos futuramente.

\section{CONCLUSÕES}

Há uma ampla oferta de dentifrícios infantis disponíveis para a comercialização de diferentes marcas, composições e preços. Ao analisar as embalagens primárias e secundárias dos produtos, observou-se que, em sua maioria, elas se encontravam de acordo com as normas estabelecidas pelas Resoluções de $n^{\circ}$ 79/2000 e n 7/2015 da Anvisa. Contudo, não há padronização das recomendações dos fabricantes em relação ao modo de uso desses produtos para a garantia do máximo efeito da ação do fluoreto no controle da cárie dentária com o mínimo risco para o desenvolvimento de fluorose dental.
Atualmente, não existe no Brasil uma legislação vigente que determine a quantidade mínima de concentração de $F$ solúvel nos dentifrícios infantis ao longo do seu prazo de validade, ainda que as evidências científicas demonstrem que dentifrícios com concentrações de $\mathrm{F}$ menores que 1.000 ppm não produzam redução significativa na incidência de cárie dentária. Além disso, é importante ressaltar que, embora todos os dentifrícios avaliados apresentassem informações sobre a não ingestão do produto e alertassem sobre a necessidade de que este fosse aplicado por um adulto ou sob sua supervisão, não há atualmente no Brasil normativas que exijam a presença de informações sobre o potencial de toxicidade dos dentifrícios fluoretados em suas embalagens.

Tendo em vista que a alta prevalência de cárie dentária, ainda é uma realidade em muitas crianças de diferentes regiões do país, é essencial a garantia de acesso aos dentifrícios fluoretados para que seja possível a manutenção dos hábitos de higiene bucal e o controle da doença. Nesse sentido, pondera-se que os consumidores devem estar atentos não somente aos preços, mas também ao custo-benefício desses produtos, uma vez que foram encontrados dentifrícios com a concentração ideal de $\mathrm{F}$ com preços muito menores que àqueles sem ou com baixa concentração.

Por fim, salienta-se a fundamentalidade da vigilância constante dos dentifrícios para que eles possam ser utilizados de maneira correta, efetiva e segura, equilibrando os benefícios e riscos do fluoreto no controle da cárie dentária.

\section{REFERÊNCIAS}

1. Martins RS, Macêdo JB, Muniz FWMG, Carvalho RS, Moreira MMSM. Composição, princípios ativos e indicações clínicas dos dentifrícios: uma revisão da literatura entre 1989 e 2011. J Health Sci Inst. 2012;30(3):287-91.

2. Cury JA, Tenuta LMA, Ribeiro CCC, Leme AFP. The importance of fluoride dentifrices to the current dental caries prevalence in Brazil. Braz Dent J. 2004;15(3):167-74. https://doi.org/10.1590/S0103-64402004000300001

3. Tenuta LMA, Cury JA. Fluoride: its role in dentistry. Braz Oral Res. 2010;24(Suppl.1):9-17. https://doi.org/10.1590/S1806-83242010000500003 
4. Agência Nacional de Vigilância Sanitária - Anvisa. Resolução RDC N ${ }^{\circ}$ 7, de 10 de fevereiro de 2015. Dispõe sobre os requisitos técnicos para a regularização de produtos de higiene pessoal, cosméticos e perfumes e dá outras providências. Diário Oficial União. 11 fev 2015.

5. Magalhães AC, Moron BM, Comar LP, Buzalaf MAR. Uso racional dos dentifrícios. Rev Gauch Odontol. 2011;59(4):615-25.

6. Cury JA, Caldarelli PG, Tenuta LMA. Necessity to review the brazilian regulation about fluoride toothpastes. Rev Saude Publica. 2015;49:1-7. https://doi.org/10.1590/S0034-8910.2015049005768

7. Santos APP, Nadanovsky P, Oliveira BH. A systematic review and meta-analysis of the effects of fluoride toothpastes on the prevention of dental caries in the primary dentition of preschool children. Community Dent Oral Epidemiol. 2013;41(1):1-12. https://doi.org/10.1111/j.1600-0528.2012.00708.x

8. Santos APP, Oliveira BH, Nadanovsky P. Effects of low and standard fluoride toothpastes on caries and fluorosis: systematic review and meta-analysis. Caries Res. 2013;47(5):382-90. https://doi.org/10.1159/000348492

9. Walsh T, Worthington HV, Glenny AM, Marinho VC, Jeroncic A. Fluoride toothpastes of different concentrations for preventing dental caries. Cochrane Database Syst Rev. 2019;3(3):1-239. https://doi.org/10.1002/14651858.CD007868.pub3

10. Agência Nacional de Vigilância Sanitária - Anvisa. Resolução RDC $N^{\circ} 79$, de 28 de agosto de 2000. Estabelece a definição e classificação de produtos de higiene pessoal, cosméticos e perfumes, e outros com abrangência neste contexto. Diário Oficial União. 31 ago 2000.

11. Agência Nacional de Vigilância Sanitária - Anvisa. Resolução RDC No 288, de 4 de junho de 2019. Altera a resolução RDC $N^{\circ} 7$, de 10 de fevereiro de 2015, que dispõe sobre os requisitos técnicos para produtos de higiene pessoal, cosméticos e perfumes. Diário Oficial União. 5 jun 2019.

12. Cury JA, Miranda LFB, Caldarelli PG, Tabchoury CPM. Dentifrícios fluoretados e o SUS-Brasil: o que precisa ser mudado? Tempus Actas Saude Colet. 2020;14(1):9-27. https://doi.org/10.18569/tempus.v14i1.2631
13. Caldarelli PG, Lucas BB, Silva BS. Contribuição da água e dentifrício fluoretado na prevalência de cárie e fluorose dentária: uma abordagem baseada em evidências. J Health Sci Inst. 2016;34(2):117-21.

14. Agência Nacional de Vigilância Sanitária - Anvisa. Resolução RDC № 16, de 12 de abril de 2011. Aprova o regulamento técnico Mercosul sobre lista de substâncias que os produtos de higiene pessoal, cosméticos e perfumes não devem conter exceto nas condições e com as restrições estabelecidas e dá outras providências. Diário Oficial União. 12 abr 2011.

15. Batista MDE, Valença AMG. Dentifrícios fluoretados e sua utilização em crianças. Arq Odontol. 2004;40(2):111-206.

16. Rebelo MAB, Fernandez-Alves CS, Bulcão LN, Cury JA. Benefícios e riscos dos suplementos medicamentosos fluoretados do mercado brasileiro. Vigil Sanit Debate. 2014;2(3):80-5. https://doi.org/10.3395/vd.v2i3.206

17. Ministério da Saúde (BR). Guia para recomendações do uso de fluoretos no Brasil. Brasília: Ministério da Saúde; 2009.

18. FDI General Assembly. Promoting oral health through fluoride toothpaste: adopted by the FDI general assembly: 7 september 2018, Buenos Aires, Argentina original version adopted by the FDI general assembly: november 2000, Paris, France. Int Dent J. 2019;69(1):17-8. https://doi.org/10.1111/idj.12469

19. Lippert F. An introduction to toothpaste: its purpose, history and ingredients. In: Van Loveren C, editor. Toothpastes. Basel: Karger; 2013. p.1-14.

20. Cury JA, Tenuta LMA. Evidence-based recommendation on toothpaste use. Braz Oral Res. 2014;28(esp):1-7. https://doi.org/10.1590/S1806-83242014.50000001

21. Roza PHJ, Studart LPC, Katz CRT. Características dos dentifrícios infantis disponíveis no mercado brasileiro. Arq Odontol. 2016;52(4):207-14.

22. Goldman AS, Yee R, Holmgren CJ, Benzian H. Global affordability of fluoride toothpaste. Global Health. 2008;4:1-8. https://doi.org/10.1186/1744-8603-4-7

\section{Contribuição dos Autores}

Fonseca LNM, Martins CA, Inagaki LT, Caldarelli PG - Concepção, planejamento (desenho do estudo), aquisição, análise, interpretação dos dados e redação do trabalho. Todos os autores aprovaram a versão final do trabalho. 